\title{
Cytological features of cervical well-differentiated mesonephric adenocarcinoma: Report a case with immunohistochemical findings
}

\author{
zhihua $\operatorname{lan}^{1}$, xin ma ${ }^{1}$, and Zhang $\operatorname{Jing}^{2}$ \\ ${ }^{1}$ Affiliation not available \\ ${ }^{2}$ University of South China Medical College
}

August 7, 2020

\begin{abstract}
Mesonephric adenocarcinoma (MA) is a rare tumor entity develops from mesonephric remnants.we demonstrate a well-differentiated case underdiagnosed as endometrial hyperplasia on a ThinPrep cytology test.
\end{abstract}

Cytological features of cervical well-differentiated mesonephric adenocarcinoma: Report a case with immunohistochemical findings

Running Title: Cytology of well-differentiated mesonephric adenocarcioma

Zhihua Lan $\mathrm{MD}^{1}$, Xin Ma MD ${ }^{1}$, Jing Zhang $\mathrm{MD}^{1}$

Department of Pathology, The First Affiliated Hospital of University of South China, Hengyang, China.

Correspondence

Jing Zhang, Department of Pathology, The First Affiliated Hospital of University of South China, Hengyang, China.

Email:zhangjingyou07@163.com

Key words: ThinPrep cytology, Mesonephric adenocarcinoma, Cervix.

\section{Key Clinical Message:}

A woman underdiagnosed as endometrial hyperplasia on a ThinPrep cytology test was proved to be welldifferentiated mesonephric adenocarcinoma by subsequent pathology. The lesson was that cell block was necessary for detecting ER, PR,Gata-3 and TTF-1.

As a rare tumor entity in female genital tract, mesonephric adenocarcinoma (MA) develops from mesonephric remnants in the stroma [1]. Only one article about cytological features of cervical MA is available. The mild morphology in cytology is a pitfall and a challenge [2]. In this report we demonstrate a well-differentiated case underdiagnosed on a ThinPrep Cytology Test.

A 44-year-old woman, gravida 2, para 2, had symptom of irregular red-brown vaginal bleeding for 9 months. Ultrasound showed a hyperechoic mass in the anterior uterine wall measured approximately $49 * 32 \mathrm{~mm}$. The ThinPrep cytology with Papanicolaou (Pap) stain demonstrated several tubular glands composed of overlapping cells (Figure 1A). There were individual atypical cells accompany with endocervical cells in a dirty background (Figure 1B). Additionally, some clusters of atypical cells with inconspicuous cytoplasm showed round-to-oval or slightly irregular nuclei (Figure 1C). Intraglandular content was not found. Based 
on these cytological features, we reported the case as atypical endometrial cells.Endometrial hyperplasia was considered [3].

Endometrial curettage was performed. Most of atypical cells with small-to-medium nuclei were arranged with well-differentiated tubular glands (Figure 2A). Some tubules contained eosinophilic luminal secretions. The scant and clear cytoplasm was again seen. In some areas, the stroma was hyaline degeneration. Immunohistochemical stains established that the tumor cells were diffusely positive to Gata-3, Pax- 8 and vimentin (Figure 2B, C). There was focal positive of p16 and CK7,but no expression of estrogen receptor, progesterone receptor,androgen receptor, $\alpha$-inhibin, carcinoembryonic antigen(CEA), Calretinin, CD10, P53 and thyroid transcription factor-1(TTF-1). Except that TTF-1 was negative, these attributes were consistent with mesonephric-like adenocarcinoma (ML-CA) [4]. Although the mass was located in uterine body, it might have originated from cervical mesonephric remnants. Because different origin suggested different biological features [4], it was necessary to examine the whole sample of uterus carefully.

The patient received total hysterectomy with bilateral adnexectomy combined with pelvic cavity lymph node dissection. Macroscopically, the grey-yellow mass with grey cut face measured $39^{*} 30^{*} 10 \mathrm{~mm}$ and was located in the uterine cavity and cervical canal (Figure 3A). Microscopically, mesonephric proliferation involved nearly the full circumference of the cervix (Figure 3B). In the stoma, the hyperplastic mesonephric glands were accompanied with atypical glands (Figure 3C). However, only neoplastic glands extruded into uterine cavity. Additionally, tumor cells exhibited higher density of nuclear chromatin, nucleo-cytoplasmic ratio and ki-67 proliferative index (Figure 3D). Based on these features, it was diagnosed as cervical MA.

Three architectures such as ductal, tubular and sheet-like variants have been characterized in cytology of cervical MA [2]. Additionally, papillary, glandular and tubulopapillary patterns have been reported in cytology of ML-CA [5]. Cord-like, solid, spindled, hobnail, glomeruloid, and retiform patterns have been described in histologic findings [5-6]. In our case, a few tubules with "mild" appearance and some indivivual atypical cells accompanied with some normal endocervical cells confused pathologists. In some clusters, the cells with irregular nuclear membranes were similar to atypical endometrial cells. The patterns of endocervical adenocarcinoma such as bird tail-like, strip of cells and rosette were not found [3]. Therefore, although the histological features were definite, the cytology was difficult to be diagnosed as carcinoma by morphology. Assuming no ultrasound data indicated the mass, our cytological diagnosis would delay surgical treatment. Perhaps the most important lesson to be learned was that cell block should be made using the residual material. The atypical cells with wild-type P53 expression would be positive for Gata-3 but negative for ER and PR [7]. These immunochemical features were helpful to exclude endometrial cells and confirm mesonephric lesions.

Sometimes remnants of the mesonephric duct are noted in cytology occasionally. These cuboidal cells with foamy vacuolated cytoplasm might be suspected as atypical cells such as abnormal endometrial cells [8]. However, eosinophilic material is present within the lumen in most cases [8]. In usual cervical specimen, diffusely mesonephric proliferation is not common. In our case, the florid hyperplastic mesonephric glands in the paracervical soft tissue were accompanied by neoplastic glands. The well differentiated neoplastic glands composed of overlapping cells with tall columnar appearance also projected into uterine cavity. The only immunohistochemical difference was the tumor glands possessed higher ki-67 proliferative index [9].

Distinguish between cervical mesonephric carcinoma and mesonephric-like carcinoma from corpus is important [10]. First, no mesonephric remnants have been observed in the untrine body neoplasm, while they are concomitants of the neoplasm in the cervical case. Some analyses suggest that uterine ML-ACs represent an aggressive subtype of endometrial carcinomas $[6,11]$. Second, ML-ACs usually show TTF-1 positivity which is absent in benign mesonephric remnants and malignant cervical mesonephric carcinoma [10].

In conclusion, mesonephric carcinoma has its unique and mild cytological characteristics which can be easily underdiagosed as atypical endometrium cells; therefore, knowledge of its cytology is important in order to eliminate it as a differential diagnose. Cell block with the support of immunocytochemical staining is indispensable. 


\section{AUTHOR CONTRIBUTIONS}

Zhihua Lan drafted the manuscript and analysed the literature.Xin Ma performed the cytology, routine HE staining, and immunohistochemistry.Jing Zhang contributed to the literature search and the final versionof the manuscript. All authors have read and approved the manuscript.

\section{CONFLICT OF INTEREST}

The authors declare no potential conflicts of interest.

\section{References}

[1]Silver SA, Devouassoux-Shisheboran M, Mezzetti TP, Tavassoli FA. Mesonephric adenocarcinomas of the uterine cervix: a study of 11 cases with immunohistochemical findings. Am J Surg Pathol . 2001; 25(3):379387.

[2]Nomoto K, Hayashi S, Tsuneyama K, Hori T, Ishizawa S. Cytopathology of cervical mesonephric adenocarcinoma: a report of two cases. Cytopathology . 2013;24(2):129-131.

[3]Wilbur DC,Chhieng DC,Guidos B,Mody DR.Epithelial Abnormalities: Glandular. In: Nayar R, Wilbur DC, eds. The Bethesda System for Reporting Cervical Cytology: Definitions, Criteria and Explanatory Notes . 3rd ed. London, United Kingdom: Springer; 2015:206-210

[4]Liang Y, Shi H, Zhu X, Yu M, Zhang X. Mesonephric Adenocarcinoma of the Uterine Corpus: A Report on 2 Cases With Comparison to Its Cervical Counterpart. Int J Gynecol Pathol. 2020 Jan 23. doi: 10.1097/PGP.0000000000000639.

[5]Kezlarian B, Muller S, Werneck Krauss Silva V, et al. Cytologic features of upper gynecologic tract adenocarcinomas exhibiting mesonephric-likedifferentiation. Cancer Cytopathol . 2019; 127(8):521-28

[6]McFarland M, Quick CM, McCluggage WG. Hormone receptor-negative, thyroid transcription factor 1-positive uterine and ovarian adenocarcinomas: report of a series of mesonephric-like adenocarcinomas. Histopathology . 2016; 68(7):1013-1020.

[7]Howitt BE, Emori MM, Drapkin R, Gaspar C, Barletta JA, Nucci MR, McCluggage WG, Oliva E, Hirsch MS. GATA3 Is a Sensitive and Specific Marker of Benign and Malignant Mesonephric Lesions in the Lower Female Genital Tract. Am J Surg Pathol . 2015; 39(10):1411-9.

[8]Hejmadi RK, Gearty JC, Waddell C, Ganesan R. Mesonephric hyperplasia can cause abnormal cervical smears: report of three cases with review of literature. Cytopathology . 2005;16(5):240-243.

[9]Skala SL, Gregg PA, Orr JW Jr, Udager AM, Brown NA, Cho KR. Cervical Mesonephric Adenocarcinoma With Novel FGFR2 Mutation. Int J Gynecol Pathol . 2019 Jul 18. doi: 10.1097/PGP.0000000000000627.

[10]Pors J, Cheng A, Leo JM, Kinloch MA, Gilks B, Hoang L. A Comparison of GATA3, TTF1, CD10, and Calretinin in Identifying Mesonephric and Mesonephric-like Carcinomas of the Gynecologic Tract.Am J Surg Pathol . 2018; 42(12):1596-1606.

[11]Horn LC, Höhn AK, Krücken I, Stiller M, Obeck U, Brambs CE. Mesonephric-like adenocarcinomas of the uterine corpus: report of a case series and review of the literature indicating poor prognosis for this subtype of endometrial adenocarcinoma. J Cancer Res Clin Oncol . 2020; 146(4):971-983.

Figure 1 (A) A neoplastic gland composed of overlapping tumor cells (liquid-based cytology test, Papanicolaou, $\times 400)$. (B) Endocervical cells with picket fence appearance (brown arrow) and scattered tumor cells (pink arrow) in the dirty background(liquid-based cytology test, Papanicolaou, $\times 400$ ).(C)Some tumor cells with irregular nuclear membranes and coarse chromatin (black arrow, liquid-based cytology test, Papanicolaou, $\times 400)$.

Figure 2 (A) Some well-differentiated tubular glands containing eosinophilic luminal secretions(HE stain, $\times 400$ ). (B) Positive Gata-3 staining(Envision, $\times 400$ ). (C) Tumor cells positive for vimentin(Envision, $\times 400$ ). 
Figure 3 (A) The gross appearance of the mass located in the the uterine cavity and cervical canal. (B) Histology showing the hyperplastic mesonephric remnants(violet arrow) and neoplastic mesonephric glands(red arrow) very close to normal endocervical glands (blue arrow,HE stain, $\times 200$ ). (C) The hyperplastic mesonephric glands (green arrow) accompanied with neoplastic glands (black arrow) in the stroma( HE stain, $\times 400$ ). (D) Tumor glands(pink arrow) showing higher ki-67 proliferative index than hyperplastic mesonephric glands(yellow arrow, HE stain, $\times 400)$.
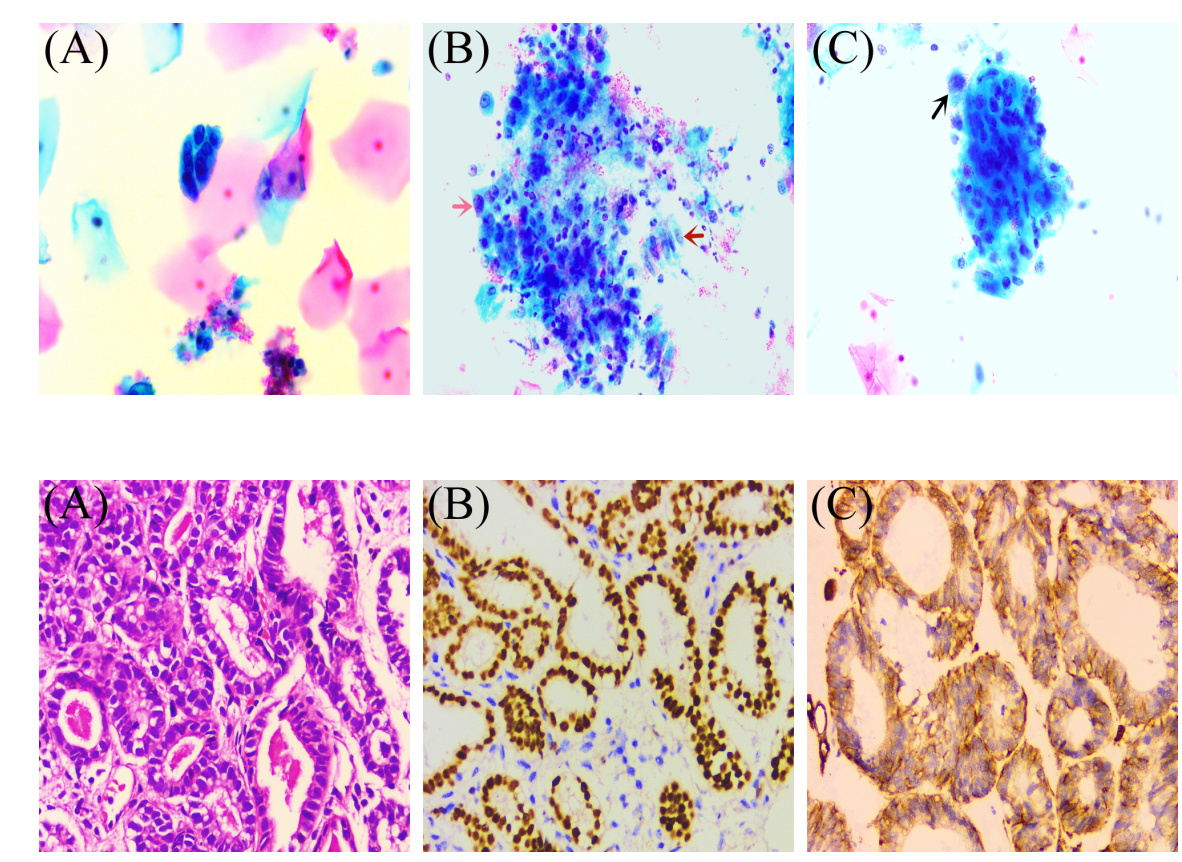

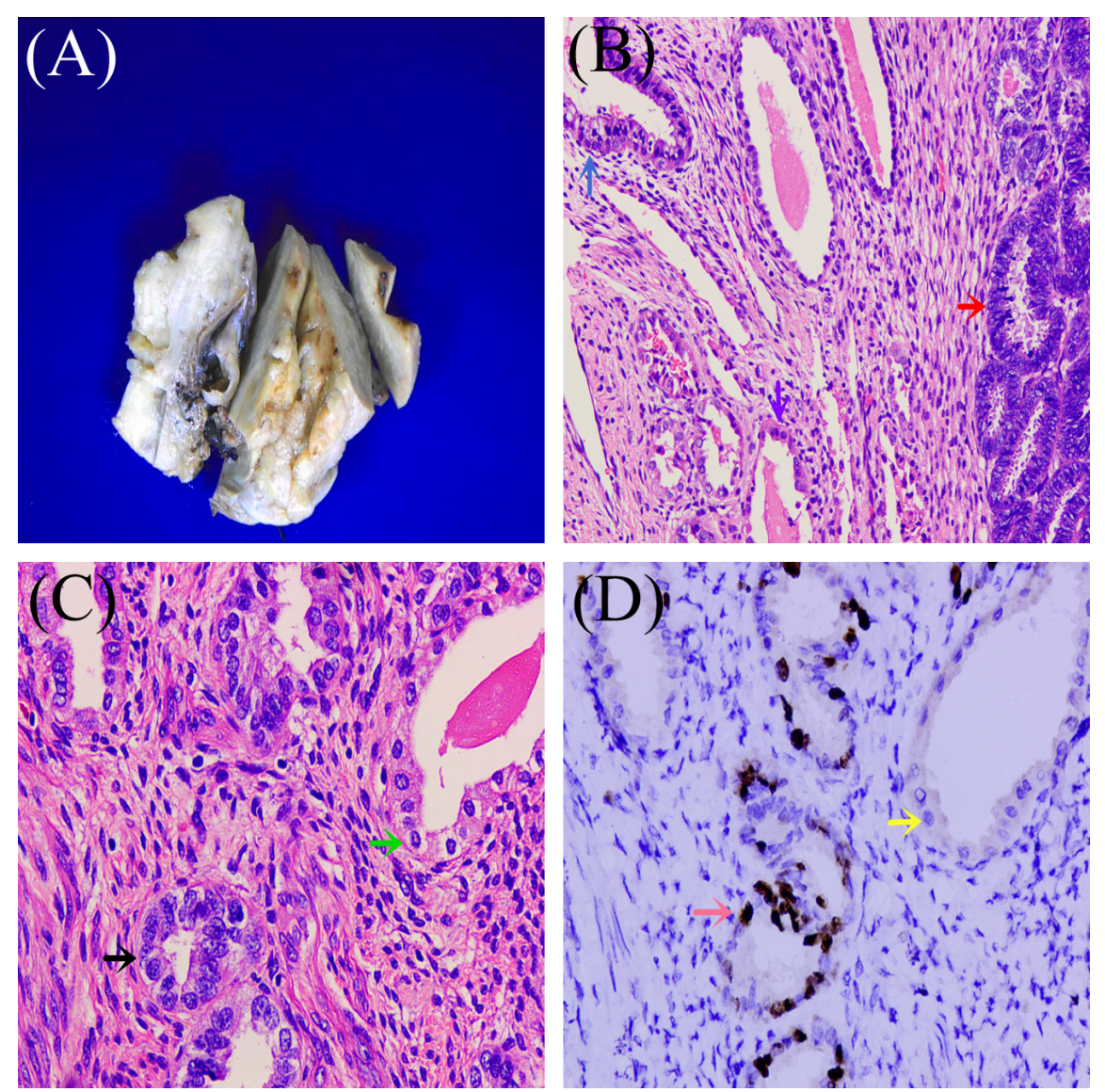